\title{
Advances in Medical Education in Anesthesia
}

\section{Multiple Choice Questions}

The multiple choice questions which appear below relate to the series of special review articles in the February 2012 issue of the Journal. For each question, select the one best response. The correct responses appear online as Electronic Supplemental Material.

1. Principles of good feedback include all of the following EXCEPT:

1) Feedback is most effective if given at the time of an event or shortly afterwards

2) Motivated recipients benefit from feedback that facilitates the learner's own reflections

3) Feedback should focus on one or two items to prevent overwhelming the learner

4) Feedback should focus on the individual, not the task

2. Which theoretical approach BEST underlies qualitative research methods in medical education?
1) Ethnography
2) Positivism
3) Epistemology
4) Participant observation

Electronic supplementary material The online version of this article (doi:10.1007/s12630-011-9641-0) contains supplementary material, which is available to authorized users.
3. Working (or short term) memory has the following characteristics EXCEPT ONE:

1) It refers to the information in our conscious memory

2) It is highly susceptible to interference

3) It has a very limited capacity

4) It is the primary mechanism for planning future goals

4. The tendency of the human brain to create inappropriate patterns on the basis of inadequate information is BEST described as:

1) Fixation error

2) Cognitive disposition to respond

3) Situation awareness

4) Latent factors

5. Medical education research is commonly described by any of the following frameworks EXCEPT:
1) The 3-P model
2) The Kirkpatrick framework
3) The transduction model
4) The Cook classification

6. Qualitative research in medical education implies all of the following EXCEPT:

1) Qualitative research aims to understand a phenomenon

2) Qualitative research requires ethics approval

3) Qualitative research relies on hypothesis testing

4) Qualitative research must be rigorous 
7. Accreditation requirements for minimal number of procedures/patients for trainees are currently guided by:
1) Empirical evidence
2) Expert consensus
3) Program averages
4) Trainee feedback

8. According to research performed in other professions, "international performance" is achieved with a minimum rehearsal time of:

1) 100 hours

2) 1,000 hours

3) 10,000 hours

4) 100,000 hours

9. Behaviourist learning theory focuses chiefly on:

1) Learning residing within the individual's memory and internal cognitive processes, such as perception, information processing, storage and retrieval.

2) Learning as an active construction of meaning brought about by the learner's experiences.

3) Positive or negative reinforcement, such the use of feedback and assessments, to bring about desired learning outcomes.

4) Learning from interacting and observing others within a social and community context.

10. Criteria for scholarship in teaching as defined by Hutchings and Shulman include all of the following EXCEPT:

1) Scholarship is in a tangible form that is publically disseminated

2) Scholarship is the subject of a research study

3) Scholarship is open to review and critique

4) Scholarship is available for others to use, build on and advance the field.

11. The Accreditation Council for Graduate Medical Education competency framework includes all of the following roles EXCEPT:

1) Interpersonal and communication skills

2) Health advocate

3) Professionalism

4) Systems based practice
12. With respect to the psychometric properties of assessments, generalizability measures which ONE of the following:

1) How well an assessment represents the construct it is intended to represent

2) How well an observed score reflects the hypothetical score if an individual is measured an infinite number of times

3) Performance relative to that of peers taking the same assessment

4) Whether an assessment is based on established content knowledge

13. Simulation-based education includes all of the following elements EXCEPT:
1) Fidelity
2) Debriefing
3) Deliberate practice
4) Simulation experience

14. Which ONE of the following simulation-based education modalities is MOST accepted for assessment?

1) Full body mannequin

2) Standardized patients

3) Part-task trainers

4) Computer based simulation

15. The CanMEDS competency framework includes all of the following roles EXCEPT:
1) Collaborator
2) Health Advocate
3) Professional
4) Educator

16. The Boyer's Model of Scholarship includes all of the following components EXCEPT:
1) Discovery
2) Application
3) Incorporation
4) Teaching 